[3] Nakano S, Morimoto S, Suzuki S, et al. Immunoregulatory role of IL-35 in $\mathrm{T}$ cells of patients with rheumatoid arthritis. Rheumatology (Oxford) 2015;54:1498-1506. doi:10.1093/rheumatology/keu528

[4] Niedbala W, Wei XQ, Cai B, et al. IL-35 is a novel cytokine with therapeutic effects against collagen-induced arthritis through the expansion of regulatory $\mathrm{T}$ cells and suppression of Th17 cells. Eur $\mathrm{J}$ Immunol 2007;37:3021-3029. doi:10.1002/eji.200737810

Acknowledgements: Grant support: APVV-15-0228 and VEGA 2/0161/16

Disclosure of Interest: None declared

DOI: 10.1136/annrheumdis-2018-eular.6522

\section{AB0065 N-CADHERIN IS DOWN-REGULATED BY DECOY RECEPTOR 3 IN SPECIFICALLY RHEUMATOID SYNOVIAL FIBROBLASTS}

K. Fukuda ${ }^{1}$, Y. Miura ${ }^{2}$, S. Hayashi ${ }^{1}$, T. Maeda ${ }^{3}$, R. Kuroda ${ }^{1} .{ }^{1}$ Department of Orthopaedic Surgery, Kobe University Graduate School of Medicine, Kobe, Japan; ${ }^{2}$ Division of Orthopedic Science, Department of Rehabilitation Science, Kobe University Graduate School of Health Sciences, Kobe, Japan; ${ }^{3}$ Department of Immunology and Rheumatolog, Stanford University, Stanford, USA

Background: Decoy receptor 3 (DcR3) is a secreted decoy tumour necrosis factor receptor and competitively binds and inhibits the TNF family including Fasligand, LIGHT, and TL1A. We previously reported that DcR3 overexpressed in rheumatoid synovial fibroblasts (RA-FLS) stimulated by TNF $\alpha$ protects the cells from Fas-induced apoptosis. ${ }^{1}$ We recently reported that DcR3 binds to TL1A expressed on RA-FLS resulting in the negative regulation of cell proliferation induced by inflammatory cytokines. ${ }^{2}$ Further, we newly revealed the gene expression profiles in RA-FLS regulated by DcR3 by using microarray data analysis. ${ }^{3}$ Among the genes in the profile, we demonstrated the possible involvement of tryptophan hydroxylase 1 down-regulated, ${ }^{4}$ interleukin 12B up-regulated by DcR3 ${ }^{5}$ and centrosomal protein $70 \mathrm{kDa}^{6}$ in the pathogenesis of $\mathrm{RA}$. The profiles also indicated that Cadherin 2/type 1/N-cadherin $(\mathrm{CDH} 2)$ was up-regulated by DcR3 (fold change 1.93). ${ }^{3} \mathrm{CDH} 2$ has been reported to be associated with cell attachment and migration, ${ }^{7}$ osteoblast differentiation, ${ }^{8}$ and the proliferation of RA-FLS. ${ }^{9}$ The hemophilic interaction of $\mathrm{CDH} 2$ suppresses the proliferation of RA-FLS through increasing the P27 ${ }^{\mathrm{Kip} 1}$ that inhibit cell-cycle progression. ${ }^{9}$

Objectives: In this study, we analysed $\mathrm{CDH} 2$ expression in RA-FLS stimulated with $\mathrm{DcR} 3$ in detail to reveal the involvement of $\mathrm{CDH} 2$ and $\mathrm{DcR} 3$ in the pathogenesis of RA.

Methods : Real-time polymerase chain reaction (real-time PCR). RA-FLS were stimulated with various concentration of DcR3-Fc or $1,000 \mathrm{ng} / \mathrm{ml}$ of $\mathrm{lgG} 1$, or left untreated in serum-free Opti-MEM for 12 hour. The relative expression levels of $\mathrm{CDH} 2$ mRNA were quantified by real-time PCR.

Immunohistochemistry.

Anti-CDH2 antibody was applied to frozen sections of synovial tissues from patients with RA or OA for overnight. After that, the expression of $\mathrm{CDH} 2$ protein was evaluated by immunohistochemical analysis.

Results: Real-time PCR demonstrated that DcR3-Fc significantly increased the expression of CDH2 mRNA in RA-FLS $(104 \%$ with $10 \mathrm{ng} / \mathrm{ml}, 112 \%$ with $100 \mathrm{ng} /$ $\mathrm{ml}$, and $200 \%$ with $1000 \mathrm{ng} / \mathrm{ml}$ ). Immunohistochemistry revealed that $\mathrm{CDH} 2$ was expressed more in the sublining layer of rheumatoid synovium than OA synovium. Conclusions: In the gene expression profiles, we focused on $\mathrm{CDH} 2$ as the gene was highly up-regulated and belonged to major functional clustering categories; protein complex assembly, cell motility, regulation of transcription, cell membrane and glycosylation. In this study, we showed that the expression of $\mathrm{CDH} 2 \mathrm{mRNA}$ in RA-FLS was induced by DcR3 and that $\mathrm{CDH} 2$ was increased in the sublining layer of rheumatoid synovium. In this study, we demonstrated that DcR3 significantly induced $\mathrm{CDH} 2$ expression in RA-FLS. Considering the fact that $\mathrm{CDH} 2$ inhibits RA-FLS proliferation, DcR3 signalling may control the hyperplasia of RA synovium.

\section{REFERENCES:}

[1] Hayashi S, et al. Arthritis Rheum. 2007;56:1067-1074

[2] Takahashi M, et al. Int J Mol Med. 2011;28:423-427.

[3] Fukuda K, et al. Int J Mol Med. 2013;32:910-916.

[4] Maeda T, et al. Mol Med Rep. 2015;12:5191-5196.

[5] Fukuda K, et al., Mol Med Rep. 2016;13:3647-3652.

[6] Fukuda K, et al. Mod Rheumatol in press.

[7] Akitaya T, et al. Dev Dyn. 1992;194:12-20.

[8] Marie P. J Cell Physiol 2002;190:297-305.

[9] Nonomura Y, et al. J Rheumatol 2009;36:698-705.

Disclosure of Interest: None declared DOI: 10.1136/annrheumdis-2018-eular.2929

\section{$\mathrm{AB} 0066$}

COMBINATION OF IL-10 AND IL-18 BUT NOT IL-6 AND IL-18 INDUCES IFN-GAMMA PRODUCTION AND SURFACE EXPRESSION OF TRAIL ON NK CELLS

K. Sato, Y. Aizaki, H. Yazawa, T. Mimura. Department of Rheumatology and Applied Immunology, Saitama Medical University, Saitama, Japan

Background: Adult-onset Still's disease (AOSD) is a systemic inflammatory disease, the cause of which is largely unknown. AOSD has been recently classified as one of the autoinflammatory diseases, in which innate rather than acquired immunity plays an important role. Serum IL-18 has been shown to be significantly high in AOSD patients.

Objectives: The aim of this study was to quantify the levels of multiple cytokines in the serum of AOSD patients, and compare the serum cytokine profile with that of healthy controls. We also attempted to evaluate the effects of the cytokines that were upregulated in the AOSD serum on natural killer (NK) cells, since NK cells are cells of innate immunity and IL-18 has been shown to enhance their cytotoxicity. ${ }^{1}$

Methods: We quantified the serum levels of 10 cytokines (IFN- $\alpha$, IFN- $\gamma$, IL-1 $\beta$, IL2, IL-4, IL-6, IL-10, IL-12p70, IL-17A and TNF- $\alpha$ ) in patients with AOSD and healthy controls using multiplex bead array assays and IL-18 using ELISA. We next sorted NK cells from peripheral blood mononuclear cells (PBMCs) of healthy controls, stimulated them in vitro, and quantified the level of IFN- $\gamma$ in the culture supernatant by ELISA and also assessed the surface expression level of tumour necrosis factor-related apoptosis-inducing ligand (TRAIL) on the cells.

Results: Compared to samples from healthy controls, the mean serum levels of IL-6 and IL-18 from AOSD patients were significantly higher. IL-10 was detectable in some of the patients. Originally, IL-18 was identified as a stimulator of IFN- $\gamma$ production $^{2}$, however, serum IFN- $\gamma$ was below the detection limit. When NK cells were stimulated in vitro with IL-18 alone, the protein level of IFN- $\gamma$ in the culture supernatant was still below the detection limit. When we further added IL- 6 and/or $\mathrm{IL}-10$ to the culture, the combination of IL-10 and IL-18, but not IL- 6 and IL-18, induced IFN- $\gamma$. As IL- 6 is a classic pro-inflammatory cytokine and IL-10 is considered to be an anti-inflammatory cytokine, this result was rather unexpected. Thus, we evaluated the expression of IL-6 and IL-10 receptors on NK cells and found that IL-10 receptors (IL-10R and IL-10R $\beta$ ) were present, but IL- 6 receptors (IL-6R and gp130) were absent. We also assessed TRAIL expression on NK cells. Here, too, the combination of IL-10 and IL-18 induced TRAIL expression the most potently. Although a previous research showed that the combination of TLR3 and IL-18 signalling synergistically induced TRAIL on NK cells in an IFN- $\gamma$ dependent manner ${ }^{3}$, the addition of an anti-IFN- $\gamma$ antibody did not diminish the TRAIL expression.
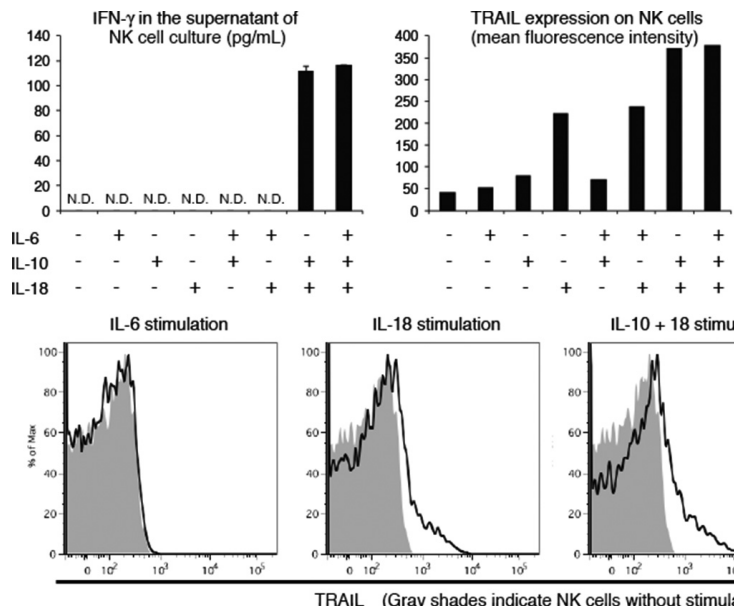

IL- $10+18$ stimulation

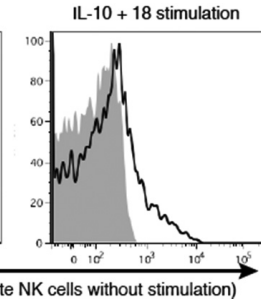

Abstract AB0066 - Figure 1

Conclusions: A combination IL-10 and IL-18, not IL-6 and IL-18, induced the production of IFN- $\gamma$ and surface expression of TRAIL in NK cells. This TRAIL expres sion did not evidently depend on IFN- $\gamma$. TRAIL was expected to be useful for the treatment of malignancy, but it turned out to be toxic to hepatocytes ${ }^{4}$. Since NK cells are rich in the liver and the abnormality of liver function is among the major symptoms in AOSD, we suggest that the combination of IL-10 and IL-18 may cause liver dysfunction by inducing TRAIL on NK cells in the liver.

\section{REFERENCES:}

[1] Akira S. Curr Opin Immunol. 2000;12:59-63.

[2] Okamura H, et al. Nature 1995;378:88-91.

[3] Tu Z, et al. Cell Immunol 2011;271:286-91.

[4] Jo M, et al. Nat Med. 2000;6:564-7. 
Disclosure of Interest: None declared

DOI: 10.1136/annrheumdis-2018-eular.3407

\section{AB0067 ALLOSTERIC RECEPTOR MODULATION OF A FREE FATTY ACID RECEPTOR TURNS NATURAL AGONISTS INTO POTENT ACTIVATORS OF THE SUPEROXIDE GENERATING NEUTROPHIL NADPH-OXIDASE}

L. Björkman, J. Mårtensson, A. Holdfeldt, H. Forsman, M. Gabl, M. Sundqvist, C. Dahlgren. Rheumatology and inflammation research, University of Gothenburg, Gothenburg, Sweden

Background: Short chain fatty acids are generated in the colon by bacterial fermentation of dietary fibres and serve as natural agonists for the free fatty acid receptor 2 (FFA2R/GPR43) which belongs to the large family of G-protein coupled receptors. We have earlier shown that acetate triggers an increase in the cytosolic concentration of free $\mathrm{Ca}^{2+}$ in neutrophils without any assembly/activation of the superoxide generating NADPH-oxidase (Mol Cell Biol. 2016 Sep 26;36 (20):2583-95). Allosteric modulators bind to receptors at sites topographically distinct from the agonist/antagonist binding site and can regulate receptor functions positively or negatively.

Objectives: We undertook this study to determine whether an FFA2R selective modulator affects the neutrophil response induced by natural FFA2R agonists.

Methods: Neutrophils were collected from healthy blood donors. Release of superoxide anions generated by the assembled/activated NADPH-oxidase was recorded by sensitive isoluminol/HRP amplified chemiluminescence method. Intracellular $\mathrm{Ca}^{2+}$ transients were measured with FURA 2-AM labelled neutrophils.

Results: The allosteric modulator lacked a direct activating effect on neutrophils, but turned natural FFA2R agonists into potent activating agonists that triggered not only a transient rise in the cytosolic concentration of free $\mathrm{Ca}^{2+}$ ions but also an assembly of the NADPH-oxidase. The NADPH-oxidase activity induced by the combined effect of the allosteric modulator and the natural agonist acetate could be further increased in neutrophils treated with the pro-inflammatory cytokine TNF- $\alpha$. The receptor selectivity was demonstrated through the inhibition of the neutrophil activity by the novel FFA2R antagonist CATPB. In addition, the allosteric modulator lacked effect on neutrophil responses triggered by a novel and selective agonist for the closely related GPR84, a receptor that recognises medium chain fatty acids.

Conclusions: Allosteric modulators that positively co-operate with natural FFA2R agonists and prime neutrophils in their response to such agonists may serve as good tools for further unravelling the physiological functions of the FFA2R and its involvement in various diseases. In this study, allosteric modulation of FFA2R is introduced as a receptor selective mechanism to prime neutrophils to produce increased amounts of reactive oxygen species.

\section{REFERENCE:}

[1] Björkman L, Mårtensson J, Winther M, Gabl M, Holdfeldt A, Uhrbom M, Bylund J, Højgaard Hansen A, Pandey SK, Ulven T, Forsman H, Dahlgren C. The Neutrophil Response Induced by an Agonist for Free Fatty Acid Receptor 2 (GPR43) Is Primed by Tumor Necrosis Factor Alpha and by Receptor Uncoupling from the Cytoskeleton but Attenuated by Tissue Recruitment. Mol Cell Biol. 2016 Sep 26;36(20):2583-95. doi:10.1128/ MCB.00161-16 [Print 2016 Oct 15].

Disclosure of Interest: None declared DOI: 10.1136/annrheumdis-2018-eular.3211

\section{AB0068 100 ALARMINS S100A8 AND S100A9 MODULATE THE INFLAMMATORY MICROENVIRONMENT IN EARLY TENDINOPATHY}

L.A.N. Crowe ${ }^{1}$, M. Akbar ${ }^{1}$, K. Patommel ${ }^{1}$, S.M. Kitson ${ }^{1}$, E. Garcia Melchor ${ }^{1}$, D S. Gilchrist ${ }^{1}$, G.A. Murrell ${ }^{2}$, I.B. Mclnnes ${ }^{1}$, N.L. Millar ${ }^{1} .{ }^{1}$ Institute of Infection, Immunity and Inflamamtion, University of Glasgow, Glasgow, UK; ${ }^{2}$ Department of Orthopaedic Surgery, St. Georges Hospital Campus, University of New South Wales, Sydney, Australia

Background: Alarmins- also referred to as damage associated molecular patterns (DAMPS)- are endogenous molecules mobilised in response to tissue damage known to activate the innate immune system in the early stages of disease. The molecular mechanisms that regulate inflammatory and remodelling pathways in tendinopathy are largely unknown therefore identifying early immune effectors is essential to understanding the pathology. S100A8 and S100A9 are constitutively expressed by cells of myeloid origin; under pathological conditions they are induced in other cell types in response to environmental triggers and cellular damage.

Objectives: Based on previous investigations we sought evidence of S100A8/A9 expression in human tendinopathy and thereafter, to explore mechanisms whereby $\mathrm{S} 100$ proteins may regulate release of inflammatory mediators and matrix synthesis in human tenocytes.

Methods: Torn supraspinatus tendon (established pathology) and intact subscapularis tendon (early pathology) biopsies were collected from patients undergoing arthroscopic shoulder surgery. Control samples of healthy hamstring tendon were collected from patients undergoing hamstring tendon $\mathrm{ACL}$ reconstruction. S100A8/A9 expression was analysed at transcript and protein level using quantitative RT-PCR and immunohistochemistry, respectively. Primary human tenocytes were cultured from hamstring tendon tissue. The in vitro effect of recombinant human $\mathrm{S} 100 \mathrm{~A} 8 / \mathrm{A} 9$ on human tenocytes was measured using quantitative RT-PCR and release of inflammatory mediators was measured at protein level by ELISA

Results: Immunohistochemical staining of tendinopathic tissues indicated the presence of S100A8 and S100A9 in tendinopathy with early diseased tissue dis playing a distinct increase in S100A8 and S100A9 expression compared with control and established pathology. These findings were mirrored by data obtained at transcript level from both early and late pathology. Treating tenocytes with exogenous S100A8/9 significantly increased release of IL-6 and CCL2; however, no alterations in genes associated with matrix remodelling were observed at a transcript level.

Conclusions: The presence of S100A8 and S100A9 in early tendinopathic lesions suggests expression is upregulated in response to cellular damage. We have confirmed the presence of S100A8, S100A9, CCL2 and IL-6 in tendinopathy and propose that S100A8 and S100A9 participate in early pathology by modulating the stromal microenvironment and influencing the inflammatory profile of tenocytes. S100A8 and S100A9 may participate in a positive feedback mechanism involving enhanced leukocyte recruitment and release of pro-inflammatory cytokines from tenocytes that perpetuates the inflammatory response within the tendon in the early stages of disease. This, in turn, may contribute aberrant matrix remodelling and associated morphological deficiencies within the tendon. We pro pose S100A8 and S100A9 are active alarmins in early tendinopathy that indirectly influence matrix remodelling by perpetuating the stromal inflammatory environ ment. Selective targeting of DAMP signalling may offer novel therapeutic approaches in the management of human tendon disorders.

\section{REFERENCE:}

[1] Millar NL, et al. MicroRNA29a regulates IL-33-mediated tissue remodelling in tendon disease. Nat Commun 2015;6:6774.

Disclosure of Interest: None declared

DOI: 10.1136/annrheumdis-2018-eular.7019

\section{AB0069 INCREASED EXPRESSION OF SOLUBLE MIC-A IN THE SYNOVIAL FLUID OF RHEUMATOID ARTHRITIS} PATIENTS

L. Bernardi ${ }^{1,2}$, A. Mariotte ${ }^{1}$, A. Scanu ${ }^{2}$, D. Noël ${ }^{3}$, L. Punzi ${ }^{2}$, S. Bahram ${ }^{1}$ P. Georgel ${ }^{1}$, J. Sibilia ${ }^{1}$. 'INSERM UMR_S 1109, Fédération de Médecine Translationnelle de Strasbourg, Université de Strasbourg, Strasbourg, France; ${ }^{2}$ Rheumatology Unit, Department of Medicine-DIMED, University of Padova, Padova, Italy, ${ }^{3}$ INSERM UMR S 1183, Montpellier University, Montpellier, France

Background: MIC-A (Major histocompatibility complex class I chain-related gene $A)^{1}$ is a transmembrane or soluble protein that interacts with the activating NKG2D receptor. MIC-A stimulates effector responses mediated by NK and CD8 + T cells under cellular stress conditions, like cancer or infections. ${ }^{2}$ MIC-A is also associated with autoimmune diseases (such as rheumatic disorders) character ised by immune dysregulation triggered by environmental factors, and plays important roles in immune activation and surveillance. ${ }^{3}$ In mice, various NKG2D ligands were discovered: Rae-1, $\mathrm{H} 60$ and MULT1 families. ${ }^{4}$

Objectives: This study aims at investigating the potential pathological relevance of soluble MIC-A (sMIC-A) protein in inflammatory rheumatic diseases involving articular structures in humans. The expression of orthologous NKG2D ligands in mouse models of experimental joint inflammation is also quantified.

Methods: We collected synovial fluid (SF) from 118 subjects: 22 Rheumatoid Arthritis (RA), 13 Psoriatic Arthritis (PSOA), 12 Gout Disease (GOUT), 18 Calcium 